\title{
Heparanase regulates in vitro VEGF-C expression and its clinical significance to pancreatic ductal cell adenocarcinoma
}

\author{
$\mathrm{BIN} \mathrm{LV}^{1^{*}}, \mathrm{BIN}_{\mathrm{ZHANG}}{ }^{*}, \mathrm{XIAO-YAN} \mathrm{HU^{2 } \text { and QING-DONG ZENG }}{ }^{1}$ \\ ${ }^{1}$ Department of General Surgery, Qilu Hospital; ${ }^{2}$ Department of Cell Biology, \\ Shandong University, Jinan, Shandong 250012, P.R. China
}

Received November 13, 2014; Accepted November 24, 2015

DOI: $10.3892 / \mathrm{ol} .2016 .4085$

\begin{abstract}
Heparanase (HPSE) and vascular endothelial growth factor $\mathrm{C}$ (VEGF-C) are important cytokines that promote metastasis and angiogenesis in numerous malignant neoplasms, however, their association remains unclear in pancreatic ductal cell adenocarcinoma (PDAC). The present study aimed to investigate whether HPSE has a positive correlation with VEGF-C expression and to uncover the role it plays in the in vitro invasion of BxPC-3 cells (a pancreatic carcinoma cell line), and to analyze the value of joint detection of HPSE and VEGF-C for PDAC patients. A recombinant plasmid, GV230/HPSE was constructed and BxPC-3 cells were transiently transfected with GV230/HPSE or siRNA against HPSE. The expression levels of HPSE and VEGF-C were compared using reverse transcription quantitative PCR (RT-qPCR) and immunoblotting. The metastatic potential of treated BxPC-3 cells was evaluated using a Transwell ${ }^{\circledR}$ invasion assay. The relative mRNA levels of HPSE and VEGF-C in 34 PDAC specimens were assessed by RT-qPCR. The results of the RT-qPCR demonstrated a 10.7- and 3.24-fold elevation $(\mathrm{P}<0.01)$ of HPSE mRNA and VEGF-C mRNA, respectively, in GV230/HPSE group, whereas the HPSE siRNA group were downregulated for these mRNAs $(-2.45$-fold, $\mathrm{P}<0.01$; -1.84 -fold, $\mathrm{P}<0.01)$. The same pattern for protein expression was detected using immunoblot assays. In Transwell ${ }^{\circledR}$ invasion assays $138 \pm 5$ cells in GV230 /HPSE group and 53 \pm 4 cells in siRNA group migrated through the Matrigel ${ }^{\circledR}$. A negative correlation between the mRNA levels of HPSE and VEGF-C in PDAC specimens and the prognosis factors of the postoperative patients was identified. Spearman rank correlation analysis indicated a positive correlation between HPSE and VEGF-C in PDAC $(r=0.812, \mathrm{P}<0.01)$. HPSE regulates the expression
\end{abstract}

Correspondence to: Professor Qing-Dong Zeng, Department of General Surgery, Qilu Hospital, Shandong University, 107 West Wenhua Street, Jinan, Shandong 250012, P.R. China

E-mail: doclvbin@gmail.com; 462707177@qq.com

*Contributed equally

Key words: pancreatic ductal cell adenocarcinoma, heparanase, VEGF-C, gene overexpression, RNA interference, metastasis of VEGF-C and facilitates invasion of $\mathrm{BxPC}-3$ in vitro. Joint detection of HPSE and VEGF-C may therefore be clinically useful in determining the prognosis of pancreatic cancer patients.

\section{Introduction}

Pancreatic cancer has a high mortality rate, with the worst 1- and 5-year survival rates of all cancers. In 2015, it is estimated that there will be 48,960 new cases of pancreatic cancer and an $\sim 40,560$ individuals are expected to succumb to this disease, according to the Surveillance, Epidemiology and End Results database (1). The incidence of infiltrating pancreatic ductal cell adenocarcinoma (PDAC) is increasing, especially in age groups $>50$ years (2). Due to the late presentation of symptoms, PDAC is often diagnosed at an advanced stage. The long-term survival rates cannot be improved with radical resection. Although numerous biomarkers of PDAC are awaiting progression to the clinic, there has been a concerted effort to explore tumor markers that may be valuable in diagnosing PDAC earlier, improving prognosis, and subsequently progressing through to clinical pharmacology experiments. The approaches of the potential biomarker studies include genomics, proteomics and metabolomics (3). Identification of biomarkers with a relatively high susceptibility and specificity for PDAC is necessary.

Metastasis to distant sites in the body, via blood vessels and lymphatic ducts results in poor prognosis. Cancer cell spread is facilitated by the secretion of enzymes that can degrade the main structural elements of the basement membrane (BM) and extracellular cell matrix (ECM). Heparin sulfate proteoglycans (HSPGs) are an important constituent of the BM, and these interact with collagen IV, laminin, and numerous cytokines, chemokines, and growth factors. Heparanase (HPSE) is often secreted by cancer cells, and degrades the heparin sulfate (HS) chain of HSPGs, thus damaging the anionic and mechanical barriers surrounding the cells and promoting the proteolysis of cytokines, including members of the vascular endothelial growth factor (VEGF) family (4). The VEGF family consists of VEGFs-A-E, placental growth factor and snake venom VEGF. VEGF-A predominantly promotes angiogenesis through interactions with VEGF receptor-1,2 (VEGFR-1,2), whereas VEGF-C induces lymphangiogenesis by attaching to VEGF receptor-3 (VEGFR-3) (5). 
PDAC is known to be predominantly hypovascular and its blood flow volume reaches only one-third of the pancreatic tissues of a healthy organ $(6,7)$. Lymph node metastasis is an independent risk factor of PDAC. VEGF-C is of importance in increasing lymphatic vessel density (LVD), and VEGFR-3 expression is highly restricted to lymphatic endothelial cells $(8,9)$. On the basis of these previous studies, it seems that VEGF-C has a greater role in PDAC than VEGF-A. For this reason, the present study has selected HPSE and VEGF-C as experimental indicators to investigate whether HPSE can regulate VEGF-C expression and its role in invasion of pancreatic carcinoma cells in vitro, and to analyze the clinicopathological characteristics of PDAC patients. The present study was approved by the ethics committee of Qilu Hospital, Shandong University (Jinan, China) and written informed consent was obtained from all patients.

\section{Materials and methods}

Cell culture and HPSE expression vector construction. Human primary pancreatic adenocarcinoma BxPC-3 cells were purchased from American Type Culture Collection (Manassas, VA, USA) and maintained in Dulbecco's modified Eagle's medium (DMEM; Thermo Fisher Scientific, Inc., Waltham, MA, USA) containing 10\% fetal calf serum (Gibco; Thermo Fisher Scientific, Inc.), $50 \mathrm{U} / \mathrm{ml}$ penicillin and $50 \mu \mathrm{g} / \mathrm{ml}$ streptomycin (Sigma-Aldrich, St. Louis, MO, USA). A GV230 plasmid was purchased, containing the gene for enhanced green fluorescent protein (EGFP) and the XhoI/Kpnl restriction sites (GeneChem Co., Ltd., Shanghai, China). Total RNA was isolated from BxPC-3 with TRIzol ${ }^{\circledR}$ reagent (Invitrogen; Thermo Fisher Scientific, Inc.) and then treated with RQ1 RNAse-Free DNase (2 units DNase/1 $\mu \mathrm{g}$ RNA; Promega Corporation, Sydney, Australia). Following reverse transcription of $5 \mu \mathrm{g}$ total RNA using oligo(dT) primers (Thermo Fisher Scientific, Inc.), the resulting cDNA was then amplified by polymerase chain reaction (PCR) using Pfu DNA polymerase (Thermo Fisher Scientific, Inc.) and the following HPSE primers: Forward 5'-TCCTGCGTACCTGAGGTTTG-3', and reverse 5'-CCATTCCAACCGTAACTTCTCCT-3'. The following PCR protocol was used: $95^{\circ} \mathrm{C}$ initial denaturation $3 \mathrm{~min}, 35$ cycles $\left(95^{\circ} \mathrm{C}\right.$ subsequent denaturation $45 \mathrm{sec}, 61^{\circ} \mathrm{C}$ annealing $50 \mathrm{sec}, 72^{\circ} \mathrm{C}$ extension $3 \mathrm{~min}$ ), and $72^{\circ} \mathrm{C}$ final extension $10 \mathrm{~min}$. Glyceraldehyde-3-phosphate dehydrogenase (GAPDH) was used as a reference gene, using the same PCR conditions. The PCR products were separated using $1.5 \%$ agarose gel electrophoresis and visualized with $6 \mathrm{X}$ DNA Loading Buffer (Beyotime Institute of Biotechnology, Haimen, China) under ultraviolet light. The amplified HPSE DNA fragment was purified from the corresponding band in the agarose gel and incubated with $\mathrm{XhoI} / \mathrm{Kpnl}$ at $37^{\circ} \mathrm{C}$ for $2 \mathrm{~h}$. The processed HPSE fragment was then cloned into GV230 using T4 DNA ligase (Thermo Fisher Scientific, Inc.). The recombinant plasmid was transformed into $\mathrm{DH} 5 \alpha$ (Beyotime Institute of Biotechnology) and confirmed by both PCR of the bacterial solution and enzymatic digestion. The PCR detection used the following HPSE primers: Forward 5'-CGCGTAGTGATGCCATGTAACTGAAT-3', and reverse 5'-CGCTTCGATCCCAAGAAGGAATCAAC-3'. The PCR program was $95^{\circ} \mathrm{C}$ for $3 \mathrm{~min}, 30$ cycles $\left(95^{\circ} \mathrm{C} 45 \mathrm{sec} ; 59^{\circ} \mathrm{C}\right.$ $\left.45 \mathrm{sec} ; 72^{\circ} \mathrm{C} 50 \mathrm{sec}\right), 72^{\circ} \mathrm{C}$ for $6 \mathrm{~min}$.

Transient transfection and semiquantitative PCR. Three pairs of small interfering RNAs (siRNAs) targeting HPSE mRNA were designed and chemically synthesized (GenePharma Co., Ltd., Shanghai, China). These siRNA sequences are as follows: Sense 5'-GCUUCGAGUAUACCUUCAUTT-3', and anti-sense 5'-AUGAAGGUAUACUCGAAGCTT-3' for siRNA-1 (targeting the 1425-1446 encoding region of human HPSE); sense 5'-GUCCAACUCAAUGGUCUAATT-3', and anti-sense 5'-UUAGACCAUUGAGUUGGACTT-3' for siRNA-2 (targeting the 1612-1633 encoding region of HPSE); sense 5'-CUUGCCAGCUUUCUCAUAUTT-3', and anti-sense 5'-AUAUGAGAAAGCUGGCAAGTT-3' for siRNA-3 (targeting the 1704-1725 encoding region). Additionally, a negative control siRNA and siGAPDH were used as controls, and negative control-FAM was used to evaluate the transfection efficiency of siRNA. BxPC-3 cells were inoculated in 6-well plates and then transfected with the GV230/HPSE plasmid and 3 pairs of siRNAs in the presence of Lipofectamine ${ }^{\circledR} 2000$ (Thermo Fisher Scientific, Inc.) when 80-90\% confluent. Cells were divided into seven groups as follows: siRNA-1 group, siRNA-2 group, siRNA-3 group, GV230/HPSE group, GV230 group, blank control group, and siGAPDH group. Following transfection and $24 \mathrm{~h}$ of cell culture, total RNA and cDNA were prepared as described earlier. The HPSE expression status in every group was evaluated by PCR using specific primers. Semiquantitative analysis performed using the data generated from electrophoresis was carried out using Quantity One software (version 4.6.2; Bio-Rad Laboratories, Inc., Hercules, CA, USA). Utilizing the absorbance value of the HPSE band normalized to GAPDH, the siRNA with the best interference efficiency was selected for use in experiments in the present study.

Reverse transcription-quantitative PCR (RT-qPCR). The cDNA sample of each group (siRNA group, GV230/HPSE group, GV230 group, blank control group), generated by reverse transcription, was diluted to working concentration ( $1 \mu \mathrm{l}$ cDNA HPSE in $50 \mu \mathrm{l} \mathrm{dd}_{2} \mathrm{O} ; 1 \mu \mathrm{l}$ cDNA VEGF-C

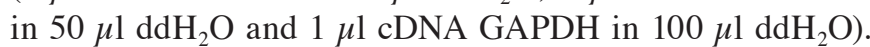
The following PCR primers were used in the present study: Forward, 5'-TCCTGCGTACCTGAGGTTTG-3', and reverse, 5'-CCATTCCAACCGTAACTTCTCCT-3' for HPSE (product, $169 \mathrm{bp}$ ); forward, 5'-ATGTGTGTCCGTCTACAGATGT-3' and reverse, 5'-GGAAGTGTGATTGGCAAAACTGA-3' for VEGF-C (product, $160 \mathrm{bp}$ ) and forward, 5'-GGAGCGAGATCCCTCCAAAAT-3' and reverse, 5'-GGCTGTTGTCATACTTCTCATGG-3' for GAPDH (product, 197 bp). RT-qPCR was performed using a Mastercycler Ep Realplex (Eppendorf, Hamburg, Germany) using SYBR Green Real-time PCR Master Mix (Toyobo Co., Ltd., Osaka, Japan) with the following settings: Initial denaturation at $95^{\circ} \mathrm{C}$, followed by 40 cycles of denaturation for $15 \mathrm{sec}$ at $95^{\circ} \mathrm{C}$, annealing for $15 \mathrm{sec}$ at $63^{\circ} \mathrm{C}$ and extension for $45 \mathrm{sec}$ at $72^{\circ} \mathrm{C}$. Melting curves were used to exclude nonspecific amplification and the emergence of primer dimers. The comparative cycle threshold method $\left(2^{-\Delta \Delta C q}\right)$ was applied to present the gene of interest relative to the internal reference gene. 

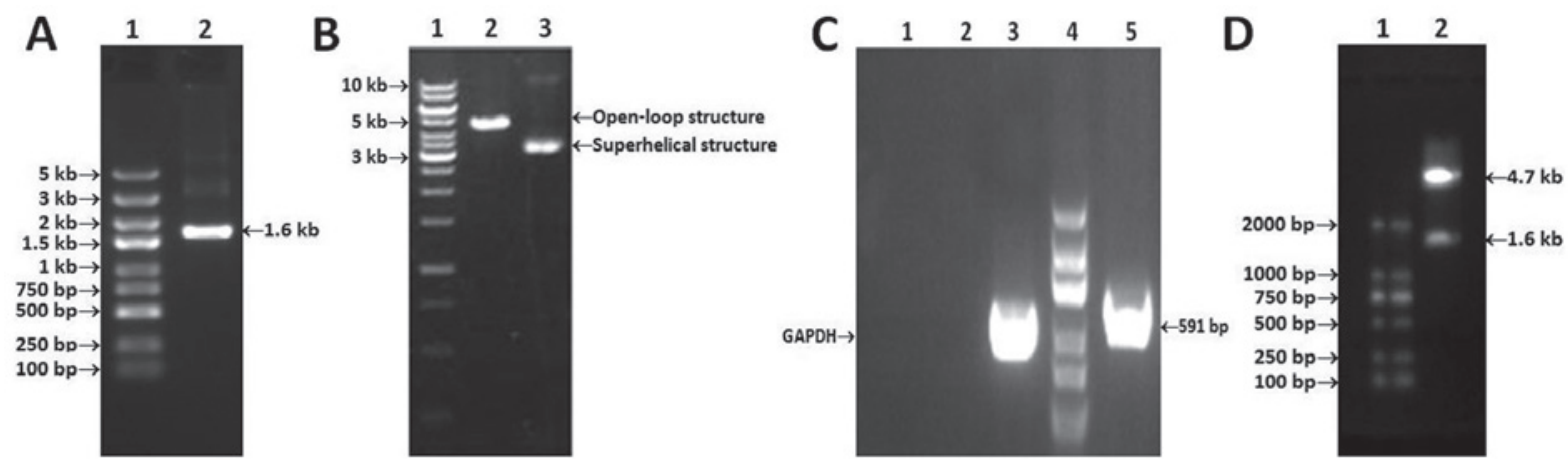

Figure 1. Construction of GV230/HPSE. (A) Electrophoresis of PCR products on 1.5\% agarose gels. Lane 1, DNA ladder marker(5 kb-100 bp; lane 2, PCR product of HPSE $(1.6 \mathrm{~kb})$. (B) Electrophoresis of GV230 vacant plasmid and product after restricted enzyme digestion. Lane 1, DNA ladder maker (10 kb-250 bp); lane 2, Linear plasmid after digestion with Xhol/Kpnl (4.7 kb); lane 3, GV230 plasmid extracted from DH5a. (C) PCR product of DNA isolated from bacterial solution. Lane 1, negative control $\left(\mathrm{ddH}_{2} \mathrm{O}\right)$; lane 2, negative control (linear plasmid self-ligation); lane 3, amplified GAPDH fragment of DH5a; lane 4, DNA ladder marker (2 kb-100 bp); lane 5, HPSE identifying band (591 bp). (D) Digestion product of recombinant plasmid GV230/HPSE. Lane 1, DNA ladder marker (2 kb-100 bp), lane 2, GV230 fragment and HPSE fragment.

Immunoblotting assay. At $48 \mathrm{~h}$ following transfection, cells were dissolved in RIPA Lysis Buffer (Beyotime Institute of Biotechnology) containing $1 \mathrm{mmol} / \mathrm{l}$ phenylmethanesulfonyl fluoride, incubated at $4^{\circ} \mathrm{C}$ for $2 \mathrm{~h}$ and then centrifuged at $14,000 \mathrm{x} \mathrm{g}$ for $20 \mathrm{~min}$. The protein concentration of the supernatant was measured using a BCA Protein Assay Kit (Thermo Fisher Scientific, Inc.). Proteins (100 mg loading) were separated by electrophoresis on $10 \%$ SDS-polyacrylamide gel and transferred to polyvinylidene fluoride membrane (PVDF; Merck Millipore, Darmstadt, Germany) by wet electroblotting (Bio-Rad Laboratories, Inc.). The PVDF membrane for each sample was incubated with rabbit monoclonal antibodies (anti-HPSE, anti-VEGF and anti- $\beta$-actin), purchased from Abcam (Cambridge, UK). $\beta$-actin was used to confirm equal loading of every sample. The secondary antibody used was horseradish peroxidase-labeled goat-anti-rabbit IgG (Sigma-Aldrich). Immunoreactive bands were visualized by enhanced chemiluminescence (Merck Millipore), exposed in the luminescent image analyzer (FluorChemE; ProteinSimple; Bio-Techne, San Jose, CA, USA) and semiquantified using Quantity One software (Bio-Rad Laboratories, Inc.).

Transwell invasion assay. Invasion assays utilized 24-well cell Transwell ${ }^{\circledR}$ inserts, coated with Matrigel ${ }^{\circledR}$ (Corning Inc., Corning, NY, USA). A total of $1 \times 10^{7}$ cells $/ \mathrm{ml}$ from each group (siRNA group, GV230/HPSE group, blank control group) suspended in $200 \mu \mathrm{l}$ DMEM (Thermo Fisher Scientific, Inc.) containing $2 \%$ fetal calf serum were seeded in the upper part of each chamber, and the lower section was filled with $500 \mu \mathrm{l}$ of complete medium. Following $48 \mathrm{~h}$ of incubation, non-invading cells on the upper surface of the Transwell ${ }^{\circledR}$ membrane $(8 \mu \mathrm{m}$ pores, polyethylene membrane) were removed with a cotton swab, and the attached cells on lower surface of the filter were fixed with methanol, stained with Giemsa and counted in at least four fields under an Olympus IX71 inverted fluorescence microscope (Olympus Corp., Tokyo, Japan). The invasion assay was performed in triplicate.

Sample collection and RT-qPCR analysis of patient tissues. 34 PDAC specimens were acquired from 20 male and 14 female patients (age range, 42-78 years; median age, 61 years) during September 2012 to December 2013 at Qilu Hospital. According to the American Joint Committee on Cancer staging system, there were 9 stage I, 11 stage II, 11 stage III, and 3 stage IV ductal cell adenocarcinomas. Among these specimens, 11 tumors were well-differentiated, 13 tumors were moderately-differentiated, and 10 tumors were poorly-differentiated. Patients were diagnosed by imaging-based findings, such as contrast-enhanced computed tomography, endoscopic ultrasonography and magnetic resonance imaging. No patients received chemotherapy or radiation therapy prior to surgery, however, percutaneous transhepatic cholangial drainage (PTCD) was allowed. Five patients underwent pancreaticoduodenectomy, 7 patients underwent pylorus-preserving pancreatoduodenectomy, 8 with distal pancreatectomy plus splenectomy, 8 with laparoscopic exploration or exploratory laparotomy and 6 with bilioenteric anastomosis or gastrojejunostomy (some with chemical impairment of celiac ganglion). Immediately following surgical removal, specimens were snap-frozen and stored in liquid nitrogen container until use. The frozen tissues were homogenized in TRIzol ${ }^{\circledR}$ reagent (Invitrogen; Thermo Fisher Scientific, Inc.), and total RNA was isolated according to the manufacturer's guideline. RT-qPCR was performed as described earlier. Pathological reports were obtained to analyze the association between prognosis factors for PDAC and HPSE and VEGF-C mRNA expression.

Statistical analysis. Results are presented as mean \pm standard error. Student's t test was used for statistical analysis of the semiquantitative PCR, $2^{-\Delta \Delta \mathrm{Cq}}$ values from RT-qPCR experiments, immunoblotting and invasion assays. The Mann-Whitney $U$ test was used to assess the correlation of HPSE and VEGF-C expression and clinicopathological parameters. Spearman rank correlation analysis was used to justify the relationship of HPSE to VEGF-C in PDAC. $\mathrm{P}<0.05$ was taken to represent a statistically significant differences in all tests.

\section{Results}

Construction of GV230/HPSE expression vector. The PCR product of HPSE and GV230 plasmid linearized by XhoI/KpnI digestion were subjected to $1.5 \%$ agarose gel electrophoresis (Fig. 1A and B). The correct recombinant plasmid was 

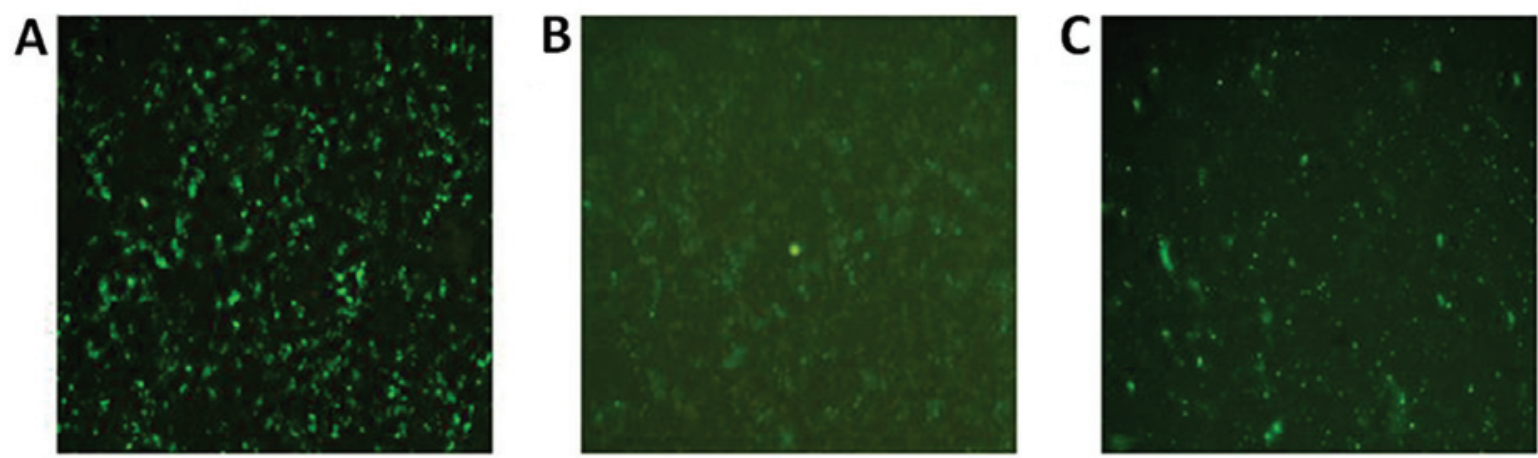

Figure 2. Transfection efficiencies, demonstrated by GFP positivity and FAM. (A) GV230, (B) GV230/HPSE and (C) siRNA-FAM.
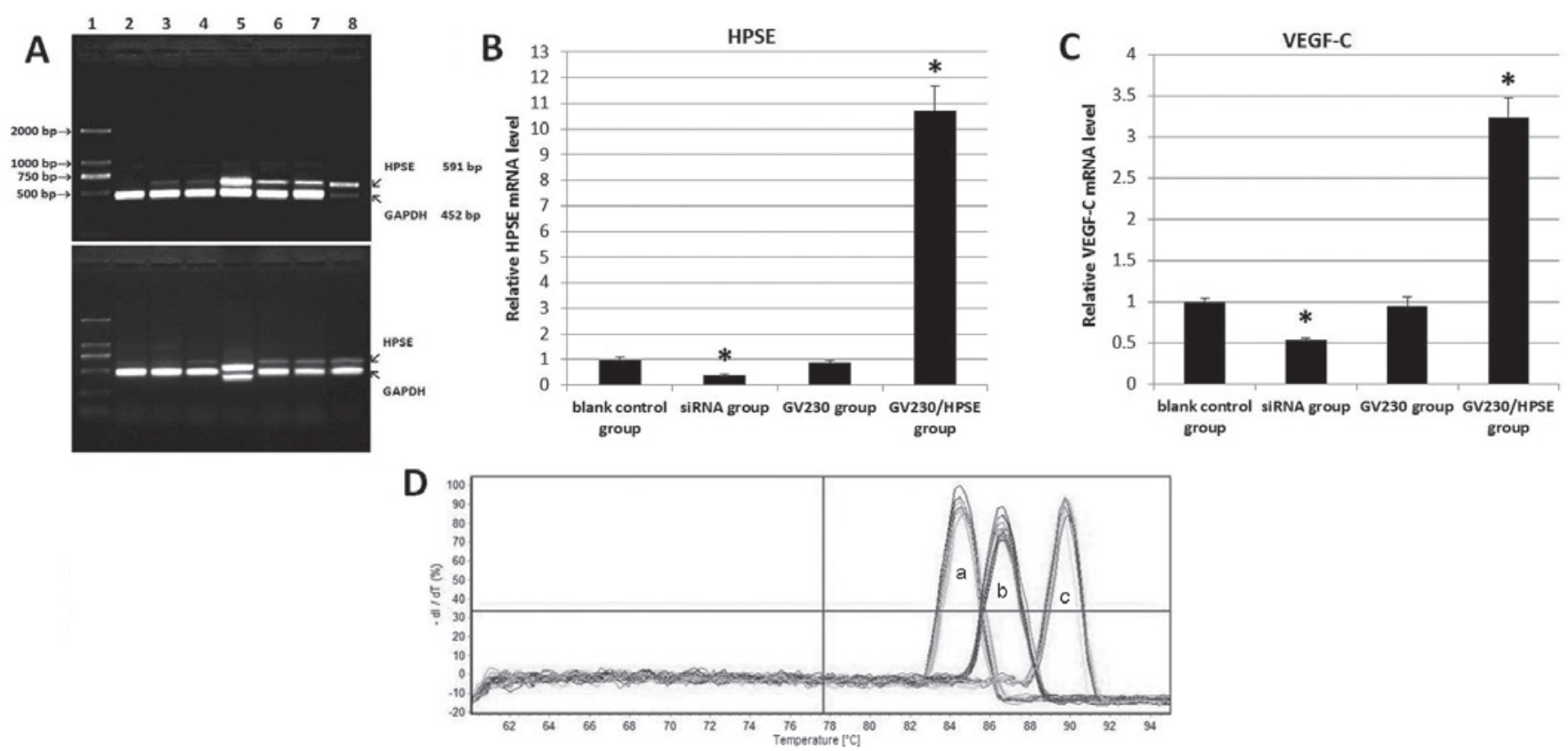

Figure 3. The influences of GV230/HPSE and HPSE-siRNAs on mRNA levels of HPSE and VEGF-C. (A) Semiquantitative PCR of HPSE and GAPDH. Two repeats of the same assay are presented. Lane 1: DNA ladder marker (2 kb-100 bp); lane 2, siRNA-1 group; lane 3, siRNA-2 group; lane 4,siRNA-3 group; lane 5, GV230/HPSE group; lane 6, GV230 group; lane 7, blank control group; lane 8, siGAPDH group. (B and C) RT-qPCR results of relative expression of HPSE and VEGF in treated BxPC-3 cells. (D) Melting curves for the RT-qPCR assays (a: HPSE, b: VEGF-C, c: GAPDH). "P<0.05. HPSE, heparanase; VEGF, vascular endothelial growth factor; siRNA, small interfering RNA; RT-qPCR, reverse transcription quantitative PCR.

confirmed by both PCR of the bacterial solution and enzymatic digestion of restriction sites (Fig. 1C and D). Furthermore, according to the sequencing report, HPSE full-length gene was successfully cloned into eukaryotic expression vector without base mutations, mismatches or frame shifts.

Transfection efficiencies of GV230, GV230/HPSE and siRNA. At $48 \mathrm{~h}$ following transfection, fluorescence images (Fig. 2A-C) were captured using an Olympus IX71 inverted fluorescence microscope (Olympus Corp.). After calculating the proportion of fluorescent cells to total cells in several fields, the approximate transfection efficiencies of the GV230 plasmid, GV230/HPSE recombinant plasmid and siRNA were calculated as $46.14 \pm 3.8 \%, 33.89 \pm 6.65 \%$ and $58.06 \pm 4.3 \%$, respectively.

The influences of GV230/HPSE and HPSE-siRNAs on mRNA levels of HPSE and VEGF-C in vitro. Images of the agarose gel PCR results were acquired (Fig. 3A), and the absorbance value of the HPSE band was normalized to that of GAPDH using Quantity One software. The relative absorbancies were $0.048 \pm 0.022$ (siRNA-1 group), $0.186 \pm 0.112$ (siRNA-2 group), $0.136 \pm 0.077$ (siRNA-3 group), $0.935 \pm 0.159$ (GV230/HPSE group), $0.448 \pm 0.047$ (GV230 group) and $0.441 \pm 0.101$ (blank control group). The siRNA-1 with the best interference effect was selected for use in subsequent trials. BxPC-3 cells were divided into four groups: siRNA group, GV230/HPSE group, GV230 group, blank control group. RT-qPCR was performed and the $2^{-\Delta \Delta \mathrm{Cq}}$ value of each sample was calculated relative to the blank control, to reflect the relative gene expression levels following treatment (Fig. 3B and C) $(10,11)$. It was ascertained that no primer dimerisation and non-specific amplification occurred, according to melting curves (Fig. 3C and D). As shown in Table I, there was a 10.7- and 3.24-fold (All P<0.01) elevation of HPSE mRNA and VEGF-C mRNA in GV230/HPSE group, respectively, whereas siRNA group exhibited decreased mRNA expression (-2.45-fold, $\mathrm{P}<0.01 ;-1.84$-fold, $\mathrm{P}<0.01)$. 
Table I. Comparison of relative mRNA levels.

\begin{tabular}{|c|c|c|c|c|c|c|}
\hline \multirow[b]{2}{*}{ Group } & \multicolumn{3}{|c|}{ HPSE mRNA } & \multicolumn{3}{|c|}{ VEGF-C mRNA } \\
\hline & $2^{-\Delta \Delta C q}$ & $\mathrm{t}^{\mathrm{a}}$ & $\mathrm{P}^{\mathrm{a}}$ & $2^{-\Delta \Delta C q}$ & $\mathrm{t}^{\mathrm{a}}$ & $\mathrm{P}^{\mathrm{a}}$ \\
\hline Blank control group & $1.000 \pm 0.094$ & & & $1.000 \pm 0.043$ & & \\
\hline siRNA group & $0.408 \pm 0.038$ & -8.970 & $<0.01$ & $0.542 \pm 0.016$ & -17.479 & $<0.01$ \\
\hline GV230 group & $0.892 \pm 0.065$ & -1.677 & 0.169 & $0.951 \pm 0.114$ & -0.715 & 0.514 \\
\hline GV230/HPSE group & $10.707 \pm 0.948$ & 17.642 & $<0.01$ & $3.239 \pm 0.233$ & 16.344 & $<0.01$ \\
\hline
\end{tabular}

${ }^{a}$ Calculated against blank control using the independent sample t-test. HPSE, heparanase; VEGF, vascular endothelial growth factor; mRNA, messenger RNA; siRNA, small interfering RNA.

Table II. Correlation between mRNA levels and clinicopathological characteristics.

\begin{tabular}{|c|c|c|c|c|c|}
\hline $\begin{array}{l}\text { Clinicopatheological } \\
\text { characteristic }\end{array}$ & Samples, no. & $\operatorname{HPSE}\left(2^{-\Delta \Delta \mathrm{Cq}}\right)$ & $\mathrm{P}_{\mathrm{HPSE}}{ }^{\mathrm{a}}$ & VEGF-C $\left(2^{-\Delta \Delta C q}\right)$ & $\mathrm{P}_{\text {VEGF-C }}{ }^{\mathrm{a}}$ \\
\hline \multicolumn{6}{|l|}{ Lymph node metastasis } \\
\hline No & 12 & $0.0594 \pm 0.0299$ & $<0.01$ & $0.0097 \pm 0.0059$ & $<0.01$ \\
\hline Yes & 22 & $0.1336 \pm 0.0587$ & $<0.01$ & $0.0334 \pm 0.0157$ & $<0.01$ \\
\hline \multicolumn{6}{|l|}{ Pathology staging } \\
\hline Well differentiated & 11 & $0.0668 \pm 0.0279$ & $<0.01$ & $0.0119 \pm 0.0058$ & $<0.01$ \\
\hline Moderately differentiated & 13 & $0.0977 \pm 0.0531$ & $<0.01$ & $0.0262 \pm 0.0100$ & $<0.01$ \\
\hline Poorly differentiated & 10 & $0.1455 \pm 0.0496$ & $<0.01$ & $0.0346 \pm 0.0151$ & $<0.01$ \\
\hline \multicolumn{6}{|l|}{ TNM staging } \\
\hline Stage I and II & 20 & $0.0656 \pm 0.0372$ & $<0.01$ & $0.0136 \pm 0.0064$ & $<0.01$ \\
\hline Stage III and IV & 14 & $0.1672 \pm 0.0767$ & $<0.01$ & $0.0413 \pm 0.0191$ & $<0.01$ \\
\hline
\end{tabular}

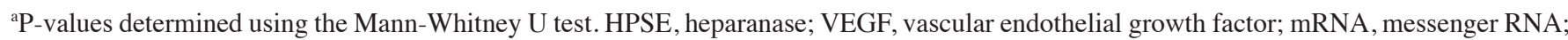
TNM, tumor-node-metastasis; Cq, quantification cycle.

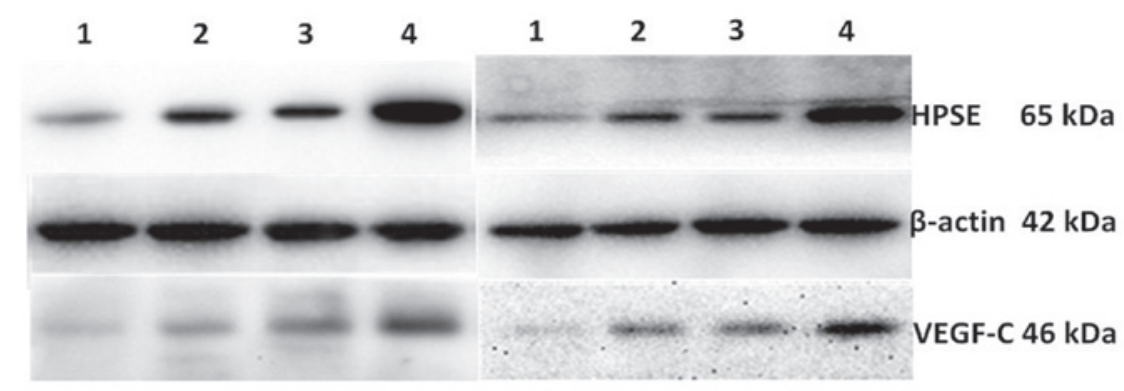

Figure 4. Expression of HPSE and VEGF-C proteins. Two repeats of the same assay are presented. Lane 1, siRNA group; lane 2, blank control; lane 3, GV230 group; lane 4, GV230/HPSE group. HPSE, heparanase; VEGF, vascular endothelial growth factor.

Expression of HPSE and VEGF-C proteins. Western blot images were acquired using FluorChemE (Fig. 4) and scanned by Quantity One to calculate the relative optical density of HPSE and VEGF bands. Due to the relatively low expression of VEGF-C and HPSE in BxPC-3, increased protein loading and exposure time were necessary and therefore increased background was visible. HPSE and VEGF-C had significantly increased expression in cells transfected with GV230/HSPE when compared to control cells $(1.245 \pm 0.077$ and $0.307 \pm 0.016$ fold respectively; $\mathrm{P}<0.01)$. Cells treated with siRNA targeted at HSPE has significantly reduced expression of HPSE and VEGF-C compared to the untreated control cells groups when compared to the blank control group $(0.161 \pm 0.024$ and $0.094 \pm 0.004$, respectively).

BxPC-3 cells invasion upon HPSE overexpression and suppression. Compared to the blank control group, $138 \pm 5$ cells in the GV230/HPSE group and $53 \pm 4$ cells in the 


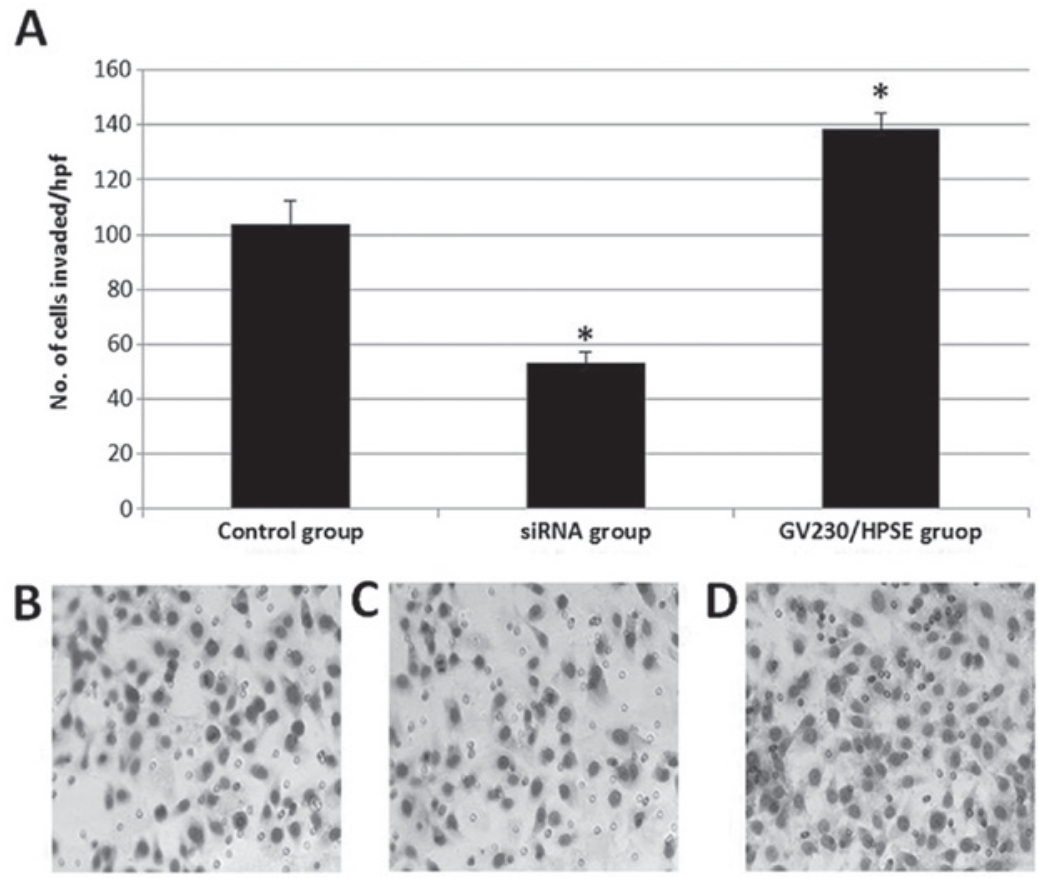

Figure 5. BxPC-3 cells invasion upon HPSE overexpression and suppression. (A) Number of cells invaded through the Matrigel. (B) Blank control group, (C) siRNA group and (D) GV230/HPSE group. "P<0.05. HPSE, heparanase; VEGF, vascular endothelial growth factor.
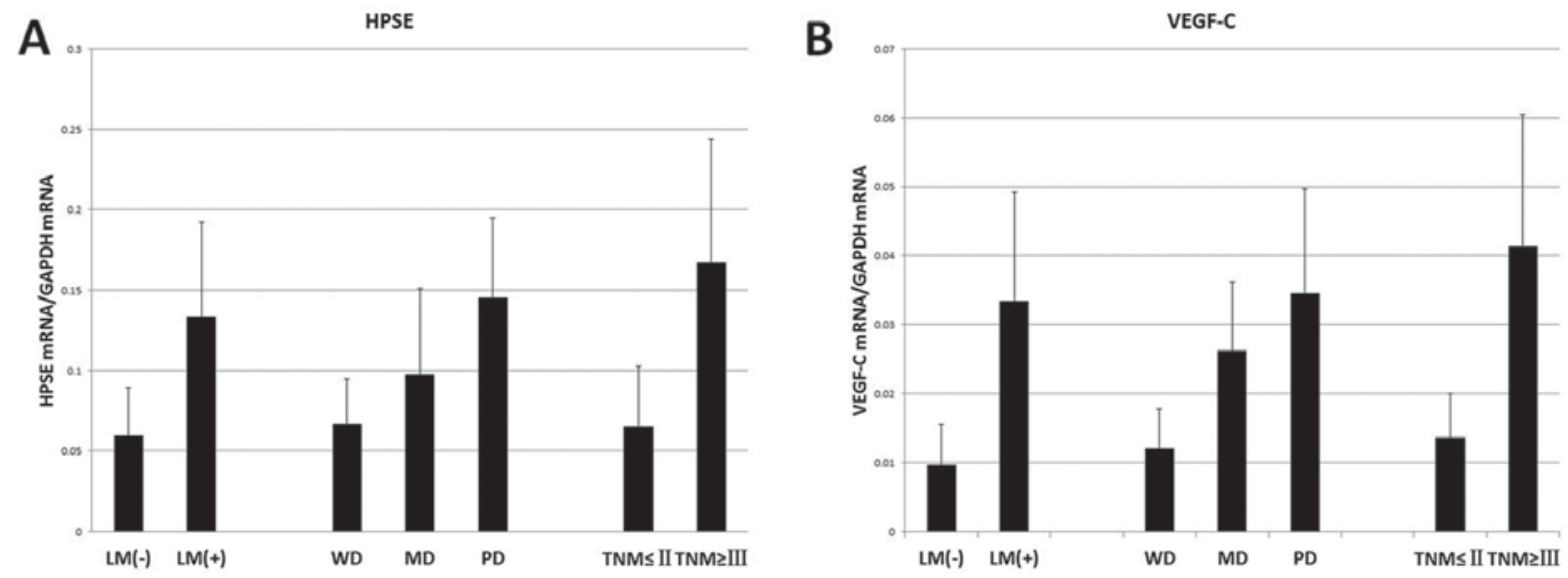

Figure 6. (A) HPSE mRNA and (B) VEGF-C mRNA expression correlated with tumor pathological characteristics. LM, lymph node metastasis; WD, well differentiated; MD, moderately differentiated; PD, poorly differentiated; TNM, tumor-node-metastasis stage; HPSE, heparanase; VEGF, vascular endothelial growth factor.

siRNA group invaded through the Matrigel and Transwell ${ }^{\circledR}$ membrane (Fig. 5A-D). This indicates that overexpression of HPSE induces BxPC-3 cell invasion, whereas knockdown of HPSE by siRNA reduces cell invasion.

Expression of HPSE and VEGF-C mRNA in human pancreatic cancer tissue and their association with prognostic factors of postoperative PDAC patients. The lymph node metastasis status, tumor differentiation grade and tumor-node-metastasis (TNM) stage were selected as the predictors of prognosis $(12,13)$. The diameter and lymphatic metastasis status of unresectable pancreatic mass were estimated by imaging tests combined with operative findings. According to Mann-Whitney U test, earlier lymphatic metastasis, poorer differentiation and more advanced tumor stages were more frequently noted in PDAC patients with high HPSE or VEGF-C expression than in patients with low HPSE or VEGF-C expression (Table II, Fig. 6). Furthermore, Spearman rank correlation analysis of $2^{-\Delta \Delta \mathrm{Cq}}$ values indicated a positive correlation between HPSE and VEGF-C $(r=0.812, \mathrm{P}<0.01)$.

\section{Discussion}

PDAC is among the leading causes of gastrointestinal cancer fatalities in China and worldwide. The 5-year survival rate is reported to be $6.8-25 \%$, and the median survival time of postoperative patients ranges from 8 to 12 months (14). Patients with lymph node metastasis have shorter survival times, with the majority of individuals surviving $\leq 2$ years. VEGF-C is expressed in several cancers, such as gastrointestinal 
malignant tumor, ovarian cancer, prostate cancer and breast cancer (15-19). Some clinical follow-up studies have associated the negative correlation of VEGF-C expression and the 5-year survival rate. Additionally, upregulation of VEGF-C is identified in those who have multiple and distant lymph node metastasis (20). These previous studies have demonstrated that HPSE, together with VEGF-C, play an essential role in the metastasis of PDAC. The present study sought to elucidate the underlying association between the two proteins using in vitro and tumor tissues experiments, including siRNA and gene overexpression techniques. The GV230 eukaryotic expression vector containing the pUC promoter and EGFP gene allowed the upregulation of HPSE and estimation of transfection efficiency. Although the GV230/HPSE vector produced less fluorescent brightness and lower transfection efficiencies compared to the empty vector, due to the recombinant size $(6.3 \mathrm{~kb})$ and the sharing of one promoter (HPSE and EGFP), this had no significant impact on the test results. Our results demonstrate a 10.7- and 3.24-fold increase of HPSE mRNA and VEGF-C mRNA, respectively, in the GV230/HPSE group, and a 2.45-and 1.84-fold decrease in siRNA group were detected by RT-qPCR. Compared to control cells, a 2.84-fold increase in HPSE protein and a 1.70-fold of VEGF-C protein was expressed in GV230 /HPSE cells, while a 2.72- and 1.91-fold reduction of proteins, respectively, were observed in the siRNA group. Thus, we conclude that HPSE modulates VEGF-C expression, although, the rangeability of HPSE is higher than VEGF-C. HPSE and other cytokines may influence VEGF-C at the same time, including components of VEGF autocrine signaling pathway $(21,22)$. Additionally, other uncontrollable factors in the present study may have weakened the regulation effect, such as the transfection efficiency and loading errors. Zetser et al (23) demonstrated that HPSE overexpression in human embryonic kidney 293, MDA-MB-435 human breast carcinoma, and rat C6 glioma cells generated a 3- to 6-fold increase in VEGF protein and mRNA levels. This increase may consist predominantly of variations in VEGF-A and VEGF-C. The recombinant plasmid used in the present study contains full length of HPSE-mRNA CDS and encodes secretory HPSE, a signal peptide (amino acids 1-35 located at the $-\mathrm{NH} 2$ terminus of the $50 \mathrm{kDa}$ subunit). The signal peptide oriented precursor protein is positioned and processed at endoplasmic reticulum and Golgi apparatus, and the resulting HPSE in its native conformation is stored in the lysosome and secreted when necessary (24). It has been previously demonstrated that elevation of VEGF in cells is associated with upregulated HPSE secretion, demonstrated by using several artificial variants of HPSE, such as deletion of the signal peptide, which generates a variant of HPSE that fails to get secreted,is resistant to proteolysis, and lacks enzymatic activity (25). Another HPSE variant is targeted to cell membrane by introducing the platelet derived growth factor receptor (PDGFR) transmembrane domain at the HPSE-COOH terminus. No significant change in VEGF expression was observed in cells expressing non-secretory HPSE, therefore VEGF upregulation requires HPSE secretion, but not its enzymatic function (24).

A number of previous studies have reported non-enzymatic activity of HPSE, as follows: i) Extrinsic addition of HPSE stimulates Akt-dependent endothelial cell migration (26); ii) extracellular signal-regulated kinase activation enhances the adhesive capability of certain cell lines, mediated by $\beta 1$-integrin, Akt and Pyk2 (27-29); iii) inducing peritumoral angiogenesis and lymphangiogenesis by regulating VEGF expression; iv) Upregulating tissue factor, and interacting with the tissue factor pathway inhibitor on the cell surface, resulting in increased endothelial and tumor cell surface coagulation activity (30). Furthermore, Zetser et al (23) provided evidence that HPSE enhanced p38 phosphorylation and is actively involved in regulation of VEGF gene expression via Src activation. Src is a cytosolic tyrosine kinase, regulated by several extracellular signal molecules and is important in the occurrence and development of tumors (31). P38 is the member of the mitogen-activated protein kinase family and participates in signaling initiated by a wide variety of extracellular stimuli. It is implicated in cell biological behaviors ranging from apoptosis, proliferation, differentiation, and to some extent, tumor cell survival and metastasis (32). We suggest that HPSE promotes lymphangiogenesis and facilitates invasion of pancreatic carcinoma cells by acting as a critical cytokine implicated in signal pathways that include VEGF-C, p38 and Src. Further trials are required to identify the HPSE receptor located in the cell membrane or cytoplasm. The metastatic potential of PDAC cells needs to be further studied in transplanted tumor models.

Based on the 34 ductal cell adenocarcinomas examined in the present study, patients with relatively high mRNA levels of HPSE and VEGF-C possess more advanced tumor stages, especially lymphatic metastasis, which is a sign of poor prognosis. In addition, a positive correlation $(r=0.812)$ demonstrates that the elevation of VEGF-C may be partly attributed to the induction of HPSE. Therefore, HPSE may serve as a novel target for cancer chemotherapy. Several HPSE inhibitors have been discovered to date, ranging from HS analogues to neutralizing antibodies. PI-88 is a chemically synthesized compound with a non-cleavable structure that can competitively inhibit HS-related angiogenesis. As a potent counterpart of HS, PI-88 exerts significant anti-tumor, anti-angiogenic, and anti-metastatic activity in numerous animal models. The activities mentioned above are attributed to intervening in HS recognition with angiogenic factors, such as VEGF and basic fibroblast growth factor, as well as suppression of HPSE activity. Other HS analogues with the same pharmacological activity, such as necuparanib and roneparstat, are already in early stage clinical development (4).

In conclusion, the present study suggests that joint detection of HPSE and VEGF-C may be of significance as a diagnostic marker and therapeutic target for patients with pancreatic cancer.

\section{Acknowledgements}

The present study was funded by the Shandong Provincial Natural Science Foundation, China (no. 2008BSB14026).

\section{References}

1. National Cancer Institute: Surveillance, Epidemiology, and End Results Program. SEER Stat Fact Sheets: Pancreas Cancer. Available at: http://seer.cancer.gov/statfacts/html/pancreas.html. Accessed April 8, 2015. 
2. Han H and Von Hoff DD: SnapShot: Pancreatic cancer. Cancer Cell 23: 424-424, 2013.

3. Winter JM, Yeo CJ and Brody JR: Diagnostic, prognostic, and predictive biomarkers in pancreatic cancer. J Surg Oncol 107: $15-22,2013$.

4. Hammond E, Khurana A, Shridhar V and Dredge K: The Role of Heparanase and Sulfatases in the Modification of Heparan Sulfate Proteoglycans within the Tumor Microenvironment and Opportunities for Novel Cancer Therapeutics. Front Oncol 4: $195,2014$.

5. Takahashi $\mathrm{H}$ and Shibuya M: The vascular endothelial growth factor (VEGF)/VEGF receptor system and its role under physiological and pathological conditions. Clin Sci (Lond) 109: 227-241, 2005.

6. Hosoki T: Dynamic CT of pancreatic tumors. AJR Am J Roentgenol 140: 959-965, 1983.

7. Matsubara H, Itoh A, Kawashima H, Kasugai T, Ohno E, et al: Dynamic quantitative evaluation of contrast-enhanced endoscopic ultrasonography in the diagnosis of pancreatic diseases. Pancreas 40: 1073-1079, 2011.

8. Omoto I, Matsumoto M, Okumura H, Uchikado Y, Setoyama T, Kita Y, Owaki T, Kijima Y, Shinchi H, Ishigami S, et al: Expression of vascular endothelial growth factor-C and vascular endothelial growth factor receptor-3 in esophageal squamous cell carcinoma. Oncol Lett 7: 1027-1032, 2014.

9. de Oliveira ATT, Reis RM, Afonso J, Martinho O, Matos D, Carvalho AL, Vazquez VL, Silva TB, Scapulatempo C Saad SS, et al: Lymphangiogenic VEGF-C and VEGFR-3 expression in genetically characterised gastrointestinal stromal tumours. Histol Histopathol 26: 1499-1507, 2011

10. Schmittgen TD and Livak KJ: Analyzing real-time PCR data by the comparative C(T) method. Nat Protoc 3: 1101-1108, 2008.

11. Bustin SA, Benes V, Garson JA, Hellemans J, Huggett J, Kubista M, Mueller R, Nolan T, Pfaffl MW, Shipley GL, et al: The MIQE guidelines: Minimum information for publication of quantitative real-time PCR experiments. Clin Chem 55: 611-622, 2009.

12. Neuzillet C, Sauvanet A and Hammel P: Prognostic factors for resectable pancreatic adenocarcinoma. J Visc Surg 148: e232-e243, 2011.

13. Argon A, Nart D, Oruc N, Coker A and Ozutemiz O: The prognostic significance of clinicopathological features and apoptosis inhibitor proteins in pancreas ductal adenocarcinoma. Acta Gastroenterol Belg 77: 229-234, 2014.

14. Jung KW, Kim MH, Lee TY, Kwon S, Oh HC, Lee SS, Seo DW and Lee SK: Clinicopathological aspects of 542 cases of pancreatic cancer: A special emphasis on small pancreatic cancer. J Korean Med Sci 22 (Suppl): S79-S85, 2007.

15. Gou HF, Chen XC, Zhu J, Jiang M, Yang Y, Cao D and Hou M: Expressions of COX-2 and VEGF-C in gastric cancer: Correlations with lymphangiogenesis and prognostic implications. J Exp Clin Cancer Res 30: 14, 2011.

16. Kozlowski M, Naumnik W, Niklinski J, Milewski R, Dziegielewski P and Laudanski J: Vascular endothelial growth factor $\mathrm{C}$ and $\mathrm{D}$ expression correlates with lymph node metastasis and poor prognosis in patients with resected esophageal cancer. Neoplasma 58: 311-319, 2011.

17. Huang KJ and Sui LH: The relevance and role of vascular endothelial growth factor $\mathrm{C}$, matrix metalloproteinase-2 and E-cadherin in epithelial ovarian cancer. Med Oncol 29: $318-323,2012$
18. Sun GG, Wang YD, Cui DW, Cheng YJ and $\mathrm{Hu}$ WN: EMP1 regulates caspase-9 and VEGFC expression and suppresses prostate cancer cell proliferation and invasion. Tumour Biol 35: 3455-3462, 2014

19. Ciobanu M, Eremia IA, Crăiţoiu S, Mărgăritescu CL, Stepan A, Pătraşcu V, Georgescu CC, Cernea D and Dumitrescu D: Lymphatic microvessels density, VEGF-C, and VEGFR-3 expression in 25 cases of breast invasive lobular carcinoma. Rom J Morphol Embryol 54: 925-934, 2013.

20. Kurahara H, Takao S, Maemura K, Shinchi H, Natsugoe S and Aikou T: Impact of vascular endothelial growth factor-C and -D expression in human pancreatic cancer: Its relationship to lymph node metastasis. Clin Cancer Res 10: 8413-8420, 2004.

21. Perrot-Applanat $M$ and Di Benedetto $M$ : Autocrine functions of VEGF in breast tumor cells Adhesion, survival, migration and invasion. Cell Adhes Migr 6: 547-553, 2012.

22. Decio A, Taraboletti G, Patton V, Alzani R, Perego P, Fruscio R, Jürgensmeier JM, Giavazzi R and Belotti D: Vascular endothelial growth factor c promotes ovarian carcinoma progression through paracrine and autocrine mechanisms. Am J Pathol 184: 1050-1061, 2014.

23. Zetser A, Bashenko Y, Edovitsky E, Levy-Adam F, Vlodavsky I and Ilan N: Heparanase induces vascular endothelial growth factor expression: Correlation with $\mathrm{p} 38$ phosphorylation levels and Src activation. Cancer Res 66: 1455-1463, 2006.

24. Zetser A, Levy-Adam F, Kaplan V, Gingis-Velitski S, Bashenko Y, Schubert S, Flugelman MY, Vlodavsky I and Ilan N: Processing and activation of latent heparanase occurs in lysosomes. J Cell Sci 117: 2249-2258, 2004.

25. Levy-Adam F, Abboud-Jarrous G, Guerrini M, Beccati D, Vlodavsky I and Ilan N: Identification and characterization of heparin/heparan sulfate binding domains of the endoglycosidase heparanase. J Biol Chem 280: 20457-20466, 2005.

26. Yuan L, Hu J, Luo Y, Liu Q, Li T, Parish CR, Freeman C, Zhu X, Ma W, Hu X, et al: Upregulation of heparanase in high-glucose-treated endothelial cells promotes endothelial cell migration and proliferation and correlates with Akt and extracellular-signal-regulated kinase phosphorylation. Mol Vis 18: 1684-1695, 2012 .

27. Riaz A, Ilan N, Vlodavsky I, Li JP and Johansson S: Characterization of heparanase-induced phosphatidylinositol 3-kinase-AKT activation and its integrin dependence. J Biol Chem 288: 12366-12375, 2013.

28. Sotnikov I, Hershkoviz R, Grabovsky V, Ilan N, Cahalon L, Vlodavsky I, Alon R and Lider O: Enzymatically quiescent heparanase augments $\mathrm{T}$ cell interactions with VCAM-1 and extracellular matrix components under versatile dynamic contexts. J Immunol 172: 5185-5193, 2004.

29. Goldshmidt O, Zcharia E, Cohen M, Aingorn H, Cohen I, Nadav L, Katz BZ, Geiger B and Vlodavsky I: Heparanase mediates cell adhesion independent of its enzymatic activity. FASEB J 17: 1015-1025, 2003.

30. Nadir Y and Brenner B: Heparanase-A Link between Coagulation, Angiogenesis, and Cancer. Rambam Maimonides Med J 3: e0002, 2012.

31. Irby RB and Yeatman TJ: Role of Src expression and activation in human cancer. Oncogene 19: 5636-5642, 2000.

32. Mittelstadt PR, Salvador JM, Fornace AJ Jr and Ashwell JD: Activating p38 MAPK: New tricks for an old kinase. Cell Cycle 4: 1189-1192, 2005. 